\title{
Spatial variation as a conventional cue*
}

\author{
N. H. FREEMAN† \\ University of Cambridge, Cambridge, England
}

Distance-discrimination studies confound the attentional response with the effective response by varying the length of the path to food. However, it is possible to use spatial variation exactly like a conventional cue by varying the length of culs at the choice point. One hundred and twenty trials were sufficient to obtain evidence of discrimination between 4 and 13 in. culs.

The behavior of rats in the early trials of training in a new maze is generally marked by exploration. This includes investigation of the cue, which is extremely difficult to quantify since the variables (of form, brightness, etc.) used in conventional cues do not elicit strong unconditioned responses. One variable which does elicit strongly is spaciousness-it elicits locomotion. Thus, if it were possible to use spatial variation like a conventional cue, then it becomes possible to observe the S's investigation of the cue during initial "adjustment." This has been accomplished in a series of studies which are being prepared for publication, and this is a report on the use of a spatial cue.

Spatial cues have generally been investigated by requiring distance discrimination, whereby the spatial extent forms a path to food (Robinson \& Wever, 1930; Greenhut, 1954). This, however, in the terminology of analyzer theory (e.g., Mackintosh, 1965), confounds cue observation with the effective response. A conventional situation would be the $S$ 's having to pass two culs to reach the choice point, the length of the culs serving as a cue to food. This was tried in a series of studies and proved to be an unusually difficult task-it took 200 correction trials to begin to teach rats to discriminate between 9 -in. and $2^{1 / 2}$-in. culs. Accordingly, the apparatus was modified to ensure choice in the presence of the cue, i.e., the choice point itself varied in area.

\section{EXPERIMENT 1 \\ Method}

The Ss were 14 female hooded rats of average weight, $160 \mathrm{~g}$. The apparatus is shown in Fig. 1. It consisted of a modified T-maze in which the choice-point area could be extended laterally by means of sliding walls. This formed the cue area, and variations in the setting of the sliding walls formed the discriminanda. The settings could be extended up to 13 in. from the closed position. The goals were provided with sunken foodcups, the lids of which retracted with a click

*Thanks are owed to A.J. Watson for help and advice. The preparation of this report was supported by the $A$. J. Wheeler Research Fellowship Fund.

+Now at the University of Durham. England. when the $S$ broke a photocell beam at the entrance to the goal alley. The end wall of one goal was painted black, the other white. The sole lighting was a 10-in. 60-W strip light, positioned $1 \mathrm{ft}$ above the perspex roof of the maze, across the cue area.

The Ss were placed on a $21^{-h}$ food-deprivation schedule with ad lib water. They were gentled in an open field for 2 weeks and were then required to learn a successive discrimination between a small cue (sliding walls, 2 in. from closed position) and a large cue (sliding walls, 13 in. from closed position). Reinforcement was two $97-\mathrm{mg}$ sugar pellets. In order to prevent "latent learning," no preadaptation in the maze was given, and this inevitably led to a high rate of refusals to run. The only concession to shaping was that both foodcups were baited on the first trial, and that choice was taken to be correct for that S. Subsequently a correction procedure was used, and training was built up to eight trials per day.

\section{Results and Discussion}

Five Ss were discarded for refusing to run; therefore, the results from nine $S$ s are reported. On the first two blocks of 40 trials, the mean scores were not significantly different from chance, by the Wilcoxon and binomial tests, at 22.2 and 20.0 , respectively. The small cue of $2 \mathrm{in}$. was made even smaller, to the closed position, and training proceeded. On the next two blocks of 40 trials, the mean scores were significantly greater than chance at $23.8(\mathrm{p}<.02$, one-tailed, binomial) and $24.9 \quad(\mathrm{p}<.02$, one-tailed, binomial). Therefore, the Ss could discriminate between 4 - and 13-in. cues in a conventional design. Indeed, on the first day of Block 3 that the smaller cue was introduced, six Ss made fewer errors, two maintained performance, and one made more errors. This is significant only at $p=.06$ by the signs test upon the hypothesis that learning will be improved. The next experiment is to train a group using the smallest cue $\mathrm{ab}$ initio.

\section{EXPERIMENT 2 \\ Method}

The Ss were 18 female hooded rats of average weight, $160 \mathrm{~g}$. The apparatus and procedure were as for
Experiment 1, only with the small cue at the closed position right from the start.

Results and Discussion

Two Ss died and 5 refused to run; therefore, the results are reported from $11 \mathrm{Ss}$. On the first two blocks of 40 trials, the scores did not differ significantly from chance, by the Wilcoxon test, with means at 19.2 $(\mathrm{SD}=2.0)$ and $18.3 \quad(\mathrm{SD}=3.2)$, respectively. On the third block of 40 trials, the scores were above chance at a mean of $23.1 \quad(\mathrm{SD}=4.4)$, with $\mathrm{p}<.05$, one-tailed, by the Wilcoxon test. The variance was transformed to $W$ (Hoel, 1960) and found to be greater than expected at $.02<\mathrm{p}<.05$. This was caused by the scores' ranging from better than chance to close to chance, with individual scores at $31,30,27,24,24$, $21,21,21,19,19,17$. Thus, the group was in the early stages of learning by the end of 120 trials. A nonparametric trend analysis after Still (1967), using a Wilcoxon test, showed improvement over the three blocks of trials at the .01 level of significance.

The Ss can discriminate spatial extents in a conventional design, and it is possible to separate the response to the cue from the effective response, exactly as in brightness or shape discrimination. A series of studies is currently being run on the effects of permitting controlled amounts of exploratory adjustment to the cues before the onset of training. REFERENCES

GREENHUT, A. Visual distance discrimination in the rat. Journal of Experimental Psychology, 1954, 47, 148-152.

HOEL, P. G. Elementary statistics. New York: Wiley, 1960.

MACKINTOSH, N. J. Selective attention in animal discrimination learning. Psychological Bulletin, 1965, 64, Psycholo.

ROBINSON, E. W., \& WEVER, E. G. Visual distance perception in the rat. University of California Publications in Psychology. 1930, 4, 233-239.

STILL, A. W. Use of orthogonal polynomials with nonparametric tests. Psychological Bulletin, 1967, 68, 327-329.

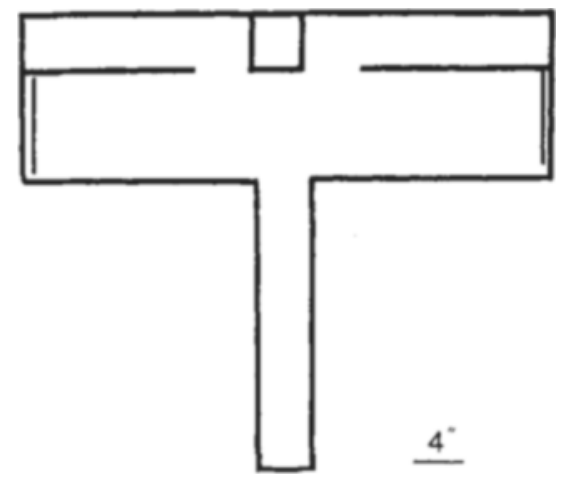

Fig. 1. Modified T-maze. Description in text. 